\title{
The coordination of joint movements during sit-to-stand motion in old adults: the uncontrolled manifold analysis
}

\author{
Masaya AnAN, PhD, $\mathrm{PT}^{1}$, Hiroka HatToRI, MS, $\mathrm{PT}^{2}$, Kenji TANIMOTO, MS, $\mathrm{PT}^{3}$, \\ Yoshio WaKimoto, MS, $\mathrm{PT}^{4}$, Takuya IbaRA, MS, $\mathrm{PT}^{5}$, Nobuhiro Kito, PhD, $\mathrm{PT}^{6}$ and \\ Koichi SHINKODA, PhD, $\mathrm{PT}^{7}$ \\ ${ }^{1)}$ Physical Therapy Course, Faculty of Welfare and Health Science, Oita University \\ ${ }^{2)}$ Kurashikiheisei Hospital \\ ${ }^{3)}$ Graduate School of Biomedical \& Health Sciences, Hiroshima University \\ ${ }^{4)}$ Graduate School of Health Sciences, Kobe University \\ ${ }^{5)}$ Kawashima Orthopaedic Hospital \\ ${ }^{6)}$ Hiroshima International University \\ ${ }^{7)}$ Department of Biomechanics, Institute of Biomedical and Health Sciences, Hiroshima University
}

\begin{abstract}
Objective: Sit-to-stand motion (STS) is a dynamic motion utilized in fundamental activities of daily living and requires extensive joint movement in the lower extremities and the trunk and coordination of multiple body segments. The present study aimed to investigate whether aging affects the motor coordination of joint movements required to stabilize the horizontal and vertical movement of center of mass using the uncontrolled manifold (UCM) analysis. Method: We recruited 39 older adults with no musculoskeletal and/or neuromuscular conditions that affected STS, along with 21 healthy younger adults. All subjects performed five STS trials from a chair with the seat height adjusted to the length of their lower leg at a selfselected motion speed. Kinematic data were collected using a three-dimensional motion analysis system. We performed the UCM analysis to assess the effects of joint angle variance (elemental variable) to stabilize the horizontal and vertical movement of COM (performance variable) and calculated the joint angle variance that does not affect COM $\left(V_{\mathrm{UCM}}\right)$, the variance that affects COM $\left(\mathrm{V}_{\text {ORT }}\right)$, and the synergy index $(\Delta V)$. Results: $\Delta \mathbf{V}$ values in the horizontal direction were higher in the older adults than in the younger adults, but $\Delta \mathbf{V}$ values in the vertical direction were lower in the older adults than in the younger adults. Conclusion: Older adults require increasing levels of stabilization of horizontal movement of COM after buttocks-off in the STS maneuver. As a result, variance in the joint angle of the lower extremities indicated no kinematic synergy for stabilizing the vertical movement of COM.
\end{abstract}

Key words: Uncontrolled manifold analysis, Sit-to-stand, Aging

(Phys Ther Res 20: 44-50, 2017)

The Sit-to-stand motion (STS) is a highly common motion that is performed as frequently as gait in daily life. It requires two tasks: transitioning the center of mass (COM) from a wide base of support (BOS) provided by the but-

Received: April 7, 2017

Accepted: September 27, 2017

Advance Publication by J-STAGE: November 30, 2017

Correspondence to: Masaya Anan, Physical Therapy Course, Faculty of Welfare and Health Science, Oita University, 700 Dannoharu, Oita 870-1192, Japan

\# e-mail: anan-masaya@ oita-u.ac.jp

doi: 10.1298/ptr.E9923 tocks, thighs, and feet to a narrow BOS provided by the feet alone, and lifting the COM from the height of sitting to that of standing. STS also represents a motion for which the muscle strength of lower extremities is particularly important ${ }^{1,2)}$. STS is associated with applied motions such as transfer and walking, and its acquisition is an essential factor to improve mobility capability. STS results from interactions characteristic of task-related, individual, and environmental constraints ${ }^{3)}$. Alexander et al. ${ }^{4)}$ reported that aging affects STS acquisition, and that older adults with difficulty performing this maneuver require prolonged STS time. We reported that older subjects who experienced dif- 
ficulty with this motion performed the STS using less energy and with more consideration for stability, relative to younger healthy subjects ${ }^{5}$.

It is important to control the redundant degrees-offreedom (DOFs), which coordinate with each body segment to accomplish tasks. STS is a whole-body movement that requires simultaneous control of many DOFs. Recently, the uncontrolled manifold (UCM) analysis has been used to quantify motor coordination. Scholz et al. ${ }^{6}$ used UCM analysis to reveal that although coordination of each segment angle stabilized the COM in the sagittal plane during STS, the head and hand postions were not stabilized. Scholz et al. ${ }^{7)}$ quantitatively showed that COM is a controlled variable of the postural system, and that its control is achieved through selective, motor equivalent changes in the joint configuration in response to support surface perturbations. The UCM analysis also revealed that the central nervous system (CNS) organizes co-variation of elemental variables to stabilize the important performance variable in a task-specific manner ${ }^{8)}$. In a previous study, STS under challenging task constraints, such as the changes in the BOS under the feet, increased the good variance that does not affect the performance variable ${ }^{9,10)}$. Greve et al. ${ }^{11)}$ reported that older adults employed greater motor flexibility, stabilizing ground reaction forces, and the horizontal and vertical movement of COM during STS. In contrast, some studies reported that, due to the inability to exploit motor abundance, older adults used a less flexible multi-joint coordination pattern to achieve the task ${ }^{12-14}$. In addition, Scholz et al. showed that joint movements did not stabilize the vertical movement of $\mathrm{COM}^{9}$. With regard to motor coordination in joint movements required to stabilize the vertical movement of COM, results have been found to vary from study to study. No published STS study has addressed the question of which joint movement contributes to the stabilization of the COM; elucidating this will require knowledge on which joint movements are coordinated.

The purpose of this study was to investigate whether aging affects the motor coordination of joint movements required to stabilize the horizontal and vertical movement of COM using the UCM analysis. We hypothesized that the motor coordination of joint movements to stabilize the COM in older adults would be decreased in both the horizontal and vertical directions. The present study clarified the differences in COM control during the STS maneuver in older adults from the perspective of motor coordination. We anticipate that this insight will lead to a better understanding of why some individuals experience difficulty with performing the STS.

\section{Method}

\section{Participants}

Thirty-nine Japanese healthy older adults registered at an employment service center (older group: 11 men and 28 women; age, $70.1 \pm 3.8$ (mean \pm SD) years; mass, $54.3 \pm 10.7$ $\mathrm{kg}$; height, $1.55 \pm 0.08 \mathrm{~m}$ ) and 21 Japanese healthy younger

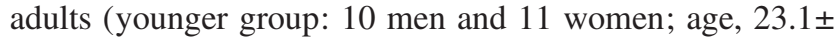
1.8 years; mass, $60.9 \pm 9.2 \mathrm{~kg}$; height, $1.67 \pm 0.09 \mathrm{~m}$ ) participated in this study. A sample size of older group and younger group achieved $80 \%$ power to detect a standardized effect size $f$ of 0.4 , maximum effect as defined by Cohen $^{15}$, and assumed a 5\% significance level in a mixed design ANOVA. Subjects were excluded if they had any history of CNS disease, lower extremity artificial joint replacement, lower extremity trauma or surgery, serious heart or lung disease, or rheumatoid arthritis. Subjects using a cane in their daily living or those who had difficulties while walking without a walking aid device were also excluded. All subjects were able to rise from a chair with the seat height adjusted to the length of their lower-leg without sitback, and had no complaints of joint pain during STS.

This study was approved by the Ethics Committee of the Division of Physical Therapy and Occupational Therapy Sciences, Graduate School of Health Sciences, Hiroshima University (1203). Subjects were given an explanation of the purpose of this study and all provided both written and oral consent prior to participation.

\section{Apparatus and experimental procedure}

Kinematic data were collected using a threedimensional motion analysis system (Vicon Motion Systems, Vicon MX) with six infrared cameras at a sampling rate of $100 \mathrm{~Hz}$ in our laboratory. Infrared-reflecting markers were attached to 40 landmarks (i.e., the temples, lateral ends of the superior nuchal line, tragi, acromia, olecranon processes, styloid processes of the ulnae, inferior edges of the last ribs, superior edges of the iliac crests, anterior superior iliac spines, posterior superior iliac spines, great trochanters, lateral and medial epicondyles of the left and right femurs, lateral and medial condyles of the left and right tibiae, lateral and medial malleoli, the first and fifth metatarsal heads, and the calcaneal tuberosities). Data were low-pass filtered using a 4th order Butterworth filter at $6 \mathrm{~Hz}$.

Subjects performed five STS trials from a chair with the seat height adjusted to the length of their lower leg. Subjects were barefoot, and the width between the feet was set as the distance between the anterior superior iliac spines. Subjects sat in a position in which the midpoints between the greater trochanter and the lateral epicondyle of the femur were aligned to the front edge of the seat. They were instructed to face forward while sitting and to fold their arms on the chest to avoid masking the reflective markers. Subjects performed the motion of rising from the initial sitting posture at a self-selected motion speed for each trial. Subjects practiced the motion sufficiently prior to measurements and there was had a rest interval after each trial. 

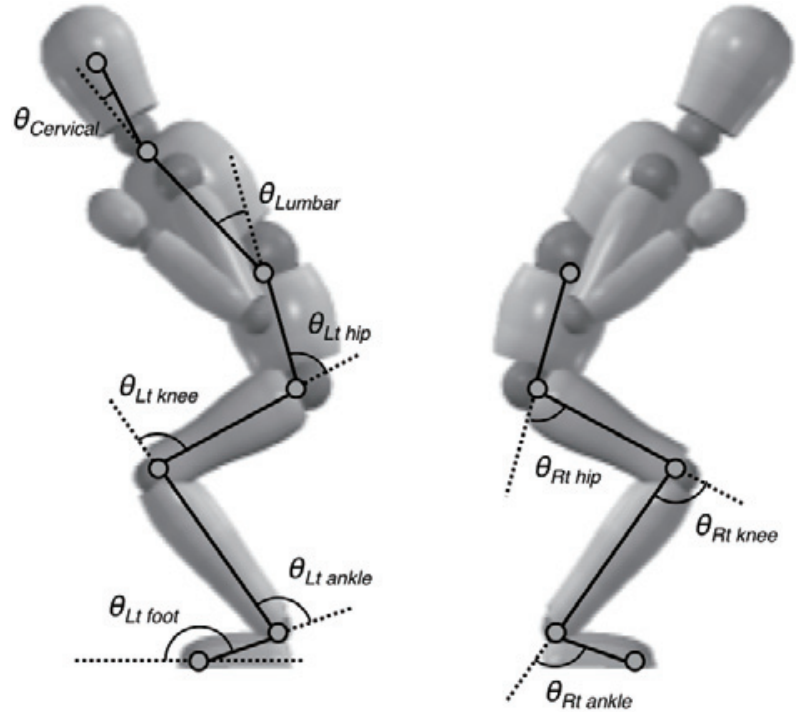

Figure 1. Stick figures showing how joint angles were computed in the sagittal plane: left foot $\left(\theta_{L t f o o t}\right)$, left ankle $\left(\theta_{\text {Lt ankle }}\right)$, left knee $\left(\theta_{L t \text { knee }}\right)$, left hip $\left(\theta_{L t ~ h i p}\right)$, right hip $\left(\theta_{R t h i p}\right)$, right knee $\left(\theta_{R t \text { knee }}\right)$, right ankle $\left(\theta_{\text {Rt ankle }}\right)$, lumbar $\left(\theta_{\text {Lumbar }}\right)$, and cervical $\left(\theta_{\text {Cervical }}\right)$.

\section{Data processing}

For data processing, processing software (Vicon Motion Systems, BodyBuilder) was used to calculate data based on collected marker coordinates. A nine-link rigidbody model of the head, thorax, pelvis, two thighs, two shanks, and two feet was constructed from the obtained marker coordinates, and the global and local coordinate systems were defined. The coordinates of each joint center were calculated according to previous studies ${ }^{16)}$. Thorax segment angle, which corresponded to the orientation of a segment relative to the global space, was differentiated to calculate the thorax angular velocity. Motion initiation was defined as the last instant of transition from negative to positive angular velocity before the occurrence of the maximum thorax angular velocity. Motion termination was defined as the first instant of transition from negative to positive angular velocity after the occurrence of the minimum thorax angular velocity. Buttocks-off was recorded using two force plates (Tec Gihan) located under the chair and was defined as the instant when the vertical vector of the ground reaction force at the buttocks was less than $10 \mathrm{~N}$. Since the forward movement of COM until buttocks-off is achieved primarily through trunk forward movement, with hardly any movement in the lower extremity joints, the present study focused on the relationship between the coordinates in the horizontal and vertical directions of COM and the joint angles after buttocks-off. And then, we extracted the data from buttocks-off to the motion termination. The extracted data were each time-normalized to align trials for computing the across-trials variance at each percentage.

Sagittal plane joint angles were calculated as the rela- tive segment angle between two adjacent segments, based on the coordinates of the center position of each of the following joints: cervical, lumbar, both hips, both knees, and both ankles. COM of the whole body was estimated at each time point using the proportion of each segment mass and the segmental length percentage projected on the sagittal plane where the center of the segment mass lies ${ }^{17)}$. The fluctuations of each joint angle and COM were computed as the variances across all trials, and means were determined for the fluctuations for both hip joints, knee joints, and ankle joints.

The UCM analysis was performed to assess the effects of joint angle variance (elemental variable) to stabilize COM (performance variable) ${ }^{18,19)}$ using data analysis software (Mathworks, MATLAB R2014a). First, a geometric model was constructed by defining the coordinates of the COM in the horizontal ( $y$ ) and vertical ( $z$ ) directions and the joint angles of left foot, both ankle, knee, hip, lumbar and cervical joints in a manner that linked them together (Fig. 1). The geometric model for COM delimited to horizontal (y) and vertical $(z)$ directions in the sagittal plane is defined by Eqs. (1) and (2), respectively:

$\mathrm{COM} y=\left(l_{\text {Ltfoot }} \cdot \cos \theta_{\text {Ltfoot }}\right) \cdot d_{L \text { tfoot }} \cdot M_{\text {Ltfoot }}+\left(l_{\text {Ltshank }} \cdot \cos \right.$ $\left.\left(\theta_{\text {Lffoot }}-\theta_{\text {Ltankle }}\right)\right) \cdot d_{\text {Ltshank }} \cdot M_{\text {Ltshank }}+\left(l_{\text {Lthigh }} \cdot \cos \left(\theta_{\text {Ltfoot }}-\theta_{\text {Ltankle }}-\right.\right.$ $\left.\left.\theta_{\text {Ltknee }}\right)\right) \cdot d_{\text {Lthigh }} \cdot M_{\text {Ltthigh }}+\left(l_{\text {Rthigh }} \cdot \cos \left(\theta_{\text {Lffoot }}-\theta_{\text {Ltankle }}-\theta_{\text {Ltknee }}-\theta_{\text {Lhip }}-\right.\right.$ $\left.\left.\theta_{\text {Rthip }}\right)\right) \cdot d_{\text {Rthigh }} \cdot M_{\text {Rthigh }}+\left(l_{\text {Rtshank }} \cdot \cos \left(\theta_{\text {Ltfoot }}-\theta_{\text {Ltankle }}-\theta_{\text {Ltknee }}-\theta_{\text {Lhip }}-\right.\right.$ $\left.\left.\theta_{\text {Rthip }}-\theta_{\text {Rtknee }}\right)\right) \cdot d_{R \text { tshank }} \cdot M_{\text {Rtshank }}+\left(l_{\text {Rtfoot }} \cdot \cos \left(\theta_{\text {Ltfoot }}-\theta_{\text {Ltankle }}-\theta_{\text {Ltknee }}-\right.\right.$ $\left.\left.\theta_{\text {Lhip }}-\theta_{\text {Rthip }}-\theta_{\text {Rtknee }}-\theta_{\text {Rtankle }}\right)\right) \cdot d_{\text {Rffoot }} \cdot M_{\text {Rffoot }}+\left(l_{\text {Pelvis }} \cdot \cos \left(\theta_{\text {Ltfoot }}-\right.\right.$ $\left.\left.\theta_{\text {Ltankle }}-\theta_{\text {Ltknee }}-\theta_{\text {Lhip }}\right)\right) \cdot d_{\text {Pelvis }} \cdot M_{\text {Pelvis }}+\left(l_{\text {Thorax }} \cdot \cos \left(\theta_{\text {Ltfoot }}-\theta_{\text {Ltankle }}-\right.\right.$ $\left.\left.\theta_{\text {Ltknee }}-\theta_{\text {Lhip }}-\theta_{\text {Lumber }}\right)\right) \cdot d_{\text {Thorax }} \cdot M_{\text {Thorax }}+\left(l_{\text {Head }} \cdot \cos \left(\theta_{\text {Ltfoot }}-\theta_{\text {Ltankle }}-\right.\right.$ $\left.\left.\theta_{\text {Ltknee }}-\theta_{\text {Lhip }}-\theta_{\text {Lumber }}-\theta_{\text {cervical }}\right)\right) \cdot d_{\text {Head }} \cdot M_{\text {Head }}$....Eq. (1)

$\operatorname{COM} z=\left(l_{L t f o o t} \cdot \sin \theta_{L \text { Lfoot }}\right) \cdot d_{L f f o o t} \cdot M_{L \text { Lfoot }}+\left(l_{L \text { Lshank }} \cdot \sin \left(\theta_{\text {Lffoot }}-\right.\right.$ $\left.\left.\theta_{\text {Ltankle }}\right)\right) \cdot d_{\text {Ltshank }} \cdot M_{\text {Ltshank }}+\left(l_{\text {Ltthigh }} \cdot \sin \left(\theta_{\text {Lffoot }}-\theta_{\text {Ltankle }}-\theta_{\text {Ltknee }}\right)\right) \cdot d_{\text {Ltthigh }}$ $\cdot M_{\text {Lthigh }}+\left(l_{\text {Rthigh }} \cdot \sin \left(\theta_{\text {Ltfoot }}-\theta_{\text {Ltankle }}-\theta_{\text {Ltknee }}-\theta_{\text {Lhip }}-\theta_{\text {Rthip }}\right)\right) \cdot d_{\text {Rtthigh }}$. $M_{\text {Rthigh }}+\left(l_{\text {Rtshank }} \cdot \sin \left(\theta_{\text {Ltfoot }}-\theta_{\text {Ltankle }}-\theta_{\text {Ltknee }}-\theta_{\text {Lhip }}-\theta_{\text {Rthip }}-\theta_{\text {Rtknee }}\right)\right)$. $d_{\text {Rtshank }} \cdot M_{\text {Rtshank }}+\left(l_{\text {Rtfoot }} \cdot \sin \left(\theta_{\text {Ltfoot }}-\theta_{\text {Ltankle }}-\theta_{\text {Ltknee }}-\theta_{\text {Lhip }}-\theta_{\text {Rthip }}-\right.\right.$ $\left.\left.\theta_{\text {Rtknee }}-\theta_{\text {Rtankle }}\right)\right) \cdot d_{\text {Rffoot }} \cdot M_{\text {Rffoot }}+\left(l_{\text {Pelvis }} \cdot \sin \left(\theta_{\text {Lffoot }}-\theta_{\text {Ltankle }}-\theta_{\text {Ltknee }}-\right.\right.$ $\left.\left.\theta_{\text {Lhip }}\right)\right) \cdot d_{\text {Pelvis }} \cdot M_{\text {Pelvis }}+\left(l_{\text {Thorax }} \cdot \sin \left(\theta_{\text {Ltfoot }}-\theta_{\text {Ltankle }}-\theta_{\text {Ltknee }}-\theta_{\text {Lhip }}-\right.\right.$ $\left.\left.\theta_{\text {Lumber }}\right)\right) \cdot d_{\text {Thorax }} \cdot M_{\text {Thorax }}+\left(l_{\text {Head }} \cdot \sin \left(\theta_{\text {Ltfoot }}-\theta_{\text {Ltankle }}-\theta_{\text {Ltknee }}-\theta_{\text {Lhip }}-\right.\right.$ $\left.\left.\theta_{\text {Lumber }}-\theta_{\text {cervical }}\right)\right) \cdot d_{\text {Head }} \cdot M_{\text {Head }}$...Eq. (2)

where $l$ is each segment length, $d$ is the COM ratio of each segment, $M$ is the mass of each segment ${ }^{20)}$, and $\theta$ is each joint angle. Therefore, the total number of elemental variables was nine.

A linearized approximation was obtained using the Jacobian matrix that relates changes in the 9 elemental variables to changes in the performance variable, as shown below:

$$
C O M-\overline{C O M}=\mathrm{J}(\Theta-\bar{\Theta}) \quad \ldots \text { Eq. }(3)
$$

where $\overline{C O M}$ is the mean COM, and $\bar{\Theta}$ is the mean of each segment angle.

The null-space of the Jacobian matrix was obtained using the mean elemental variables $\bar{\Theta}$. This space represents 
Table 1. Means \pm standard deviation of the variances of COM coordinate in the horizontal and vertical directions after buttocks-off while STS in the older and younger groups.

\begin{tabular}{llcc}
\hline & & First phase & Second phase \\
\hline COMy & Younger & $127.6 \pm 91.2$ & $184.7 \pm 164.3$ \\
& Older & $159.7 \pm 140.0$ & $179.4 \pm 121.8$ \\
$\mathrm{COM} z$ & Younger & $59.5 \pm 46.5^{*}$ & $9.4 \pm 7.0$ \\
& Older & $136.0 \pm 126.5$ & $31.0 \pm 63.5$ \\
\hline
\end{tabular}

$\left[\mathrm{mm}^{2}\right] *: \mathrm{p}<0.05$

the linear subspace of all the elemental variable deviations that do not affect the performance variable.

$$
0=J(\bar{\Theta}) \cdot \varepsilon \quad \ldots \text { Eq. }(4)
$$

The null space has $n-d$ basis vectors $\varepsilon$, where $n=9$ and $\mathrm{d}=2$ are the numbers of dimensions in the elemental variables and the performance variable, respectively. The deviations of the vector of elemental variables of each trial from the vector of the mean elemental variable across trials at each time slice were projected onto the null-space and a component perpendicular to the null space:

$$
\begin{aligned}
& \Theta_{U C M}=\sum_{i=1}^{n} \varepsilon_{i} \cdot(\Theta-\bar{\Theta}) \quad \ldots \text { Eq. }(5) \\
& \Theta_{O R T}=(\Theta-\bar{\Theta})-\Theta_{U C M} \quad \ldots \text { Eq. }(6)
\end{aligned}
$$

The variances across trials of the magnitudes of the projections were calculated, with each projection normalized to the number of dimensions in each space:

$$
\begin{aligned}
& \mathrm{V}_{U C M}=\frac{1}{N} \frac{1}{n-d} \sum_{i=1}^{N} \Theta^{2}{ }_{U C M} \quad \ldots \text { Eq. (7) } \\
& \mathrm{V}_{O R T}=\frac{1}{N} \frac{1}{d} \sum_{i=1}^{N} \Theta^{2}{ }_{O R T} \quad \ldots \text { Eq. (8) }
\end{aligned}
$$

where $\mathrm{N}$ is the number of repetitions. One component represents the variance projected onto $\mathrm{UCM}\left(\mathrm{V}_{\mathrm{UCM}}\right)$ that does not affect the performance variable ("good variance"). The other component represents the variance orthogonal to $\mathrm{UCM}\left(\mathrm{V}_{\mathrm{ORT}}\right)$ that affects the performance variable ("bad variance"). As "good variance" increases more movement patterns are used to perform a task, whereas "bad variance" increases performance variance, and thus destabilizes performance ${ }^{21)}$. Kinematic synergy is a functional structure that reduces the DOFs of movement through the coordination with each joint movement to achieve a task. If $\mathrm{V}_{\mathrm{UCM}}>\mathrm{V}_{\mathrm{ORT}}$, the selected performance variable is stabilized by kinematic synergy ${ }^{6}$. The index of synergy $\Delta \mathrm{V}$ to quantify the motor coordination was defined as follows:

$$
\begin{aligned}
& \mathrm{V}_{T O T}=\left(\frac{1}{n+d}\right)\left(d \mathrm{~V}_{O R T}+(n-d) \mathrm{V}_{U C M}\right) \quad \ldots \text { Eq. (9) } \\
& \Delta \mathrm{V}=\frac{\mathrm{V}_{U C M}-\mathrm{V}_{O R T}}{\mathrm{~V}_{T O T}} \quad \ldots \text { Eq. (10) }
\end{aligned}
$$

where $\mathrm{V}_{\text {тот }}$ is the total trial-to-trial variance. $\Delta \mathrm{V}$ was transformed using Fisher's z-transformation for statistical analy-
Table 2. Means \pm standard deviation of the variances of each joint angle after buttocks-off during STS in the older and younger groups.

\begin{tabular}{lllc}
\hline & & First phase & Second phase \\
\hline CTJ & Younger & $22.1 \pm 19.0$ & $11.2 \pm 10.7$ \\
& Older & $14.3 \pm 14.1$ & $10.3 \pm 8.64$ \\
LSJ & Younger & $11.2 \pm 8.09$ & $4.82 \pm 4.40$ \\
& Older & $10.4 \pm 15.1$ & $4.83 \pm 7.14$ \\
Hip & Younger & $16.0 \pm 12.1^{*}$ & $6.72 \pm 8.19^{*}$ \\
& Older & $36.4 \pm 31.8$ & $19.6 \pm 22.0$ \\
Knee & Younger & $8.09 \pm 7.40^{*}$ & $7.12 \pm 4.97^{*}$ \\
& Older & $25.6 \pm 28.3$ & $28.9 \pm 50.9$ \\
Ankle & Younger & $2.62 \pm 1.93^{*}$ & $1.70 \pm 0.94$ \\
& Older & $5.18 \pm 4.75$ & $6.24 \pm 9.66$ \\
\hline
\end{tabular}

$\left[\mathrm{deg}^{2}\right] *: \mathrm{p}<0.05$

sis $\left(\Delta \mathrm{V}_{\text {tran }}\right)^{22)}$. Prior to statistical analysis, we partitioned the data equally into two phases after buttocks-off (first phase and second phase). Following this, mean variances of each joint angle and COM, $\mathrm{V}_{\mathrm{UCM}}, \mathrm{V}_{\mathrm{ORT}}$, and $\Delta \mathrm{V}_{\text {tran }}$ were determined for both the first and second phases.

\section{Statistics analysis}

SPSS (23.0, IBM Japan) was used for statistical analyses. We used a mixed design ANOVA to examine the between-group factor (older vs. younger) and variance component ( $\mathrm{V}_{\mathrm{UCM}} \mathrm{VS}$. $\left.\mathrm{V}_{\mathrm{ORT}}\right)$. A significant main effect of the variance component $\left(\mathrm{V}_{\mathrm{UCM}}>\mathrm{V}_{\mathrm{ORT}}\right)$ indicated the existence of kinematic synergy. The two-sample t-test was used to compare $\mathrm{V}_{\mathrm{UCM}}, \mathrm{V}_{\mathrm{ORT}}, \Delta \mathrm{V}_{\text {tran }}$, the variances of COM coordinates in the horizontal and vertical directions, and the variances of each joint angle between the older and younger groups. The significance level was set at $\leq 5 \%$.

\section{Results}

Variance in the horizontal movement of COM in both phases did not differ significantly between the two groups, but the vertical movement of COM for the first phase was higher in the older group than in the younger group (Table $1)$. The variance in the hip and knee joint angles in both phases and the variance in the ankle joint angle for the first phase were higher in the older group than in the younger group (Table 2).

Our ANOVA showed a significant main effect of variance component $\left(\mathrm{V}_{\mathrm{UCM}}>\mathrm{V}_{\mathrm{ORT}}\right)$ in both phases for the horizontal movement, indicating the presence of kinematic synergy. No significant difference was found in $\mathrm{V}_{\text {ORT }}$ in the horizontal direction $\left(\mathrm{V}_{\text {ORT }}\right)$ between the two groups, but $\mathrm{V}_{\text {UCM }}$ in the horizontal direction $\left(\mathrm{V}_{\text {Uсм }} \mathrm{y}\right)$ for the first and second phase and $\Delta \mathrm{V}_{\text {tran }}$ in the horizontal direction $\left(\Delta \mathrm{V} y_{\text {tran }}\right)$ for the second phase were higher in the older group than in the younger group (Fig. 2a-c). $\mathrm{V}_{\mathrm{UCM}}$ and $\mathrm{V}_{\mathrm{ORT}}$ in the vertical di- 


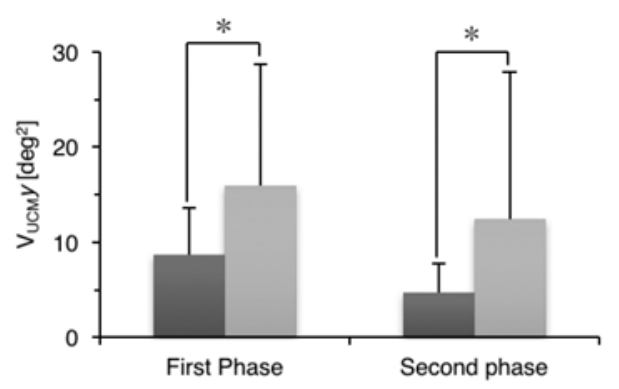

(a) $V_{\text {UCM }} y$

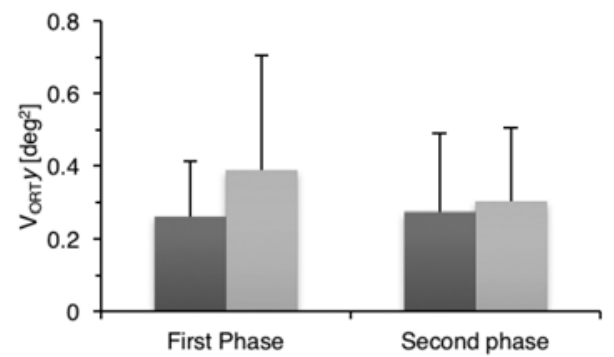

(b) $V_{\text {ORT }} y$

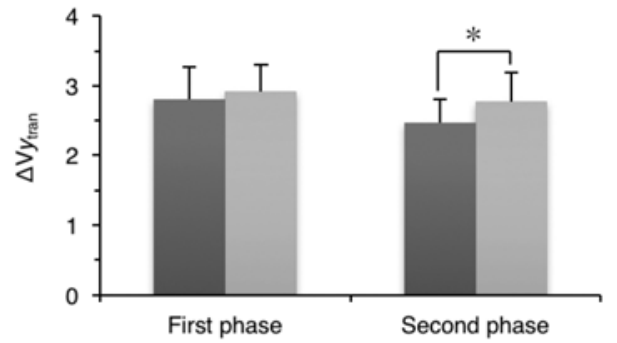

(c) $\Delta V y_{\text {tran }}$

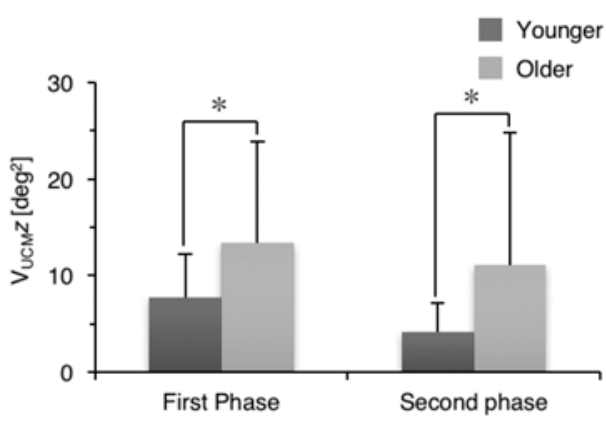

(d) $\mathrm{V}_{\mathrm{UCM}} \mathrm{z}$

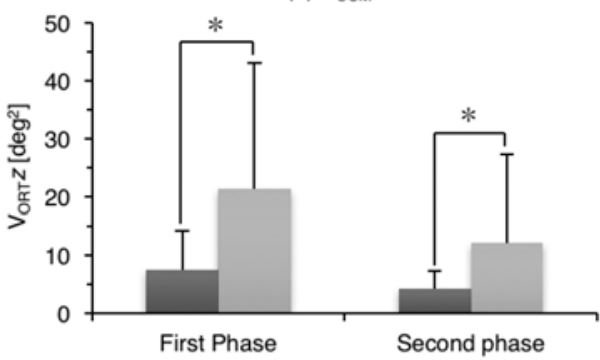

(e) $V_{\text {ORT }} z$

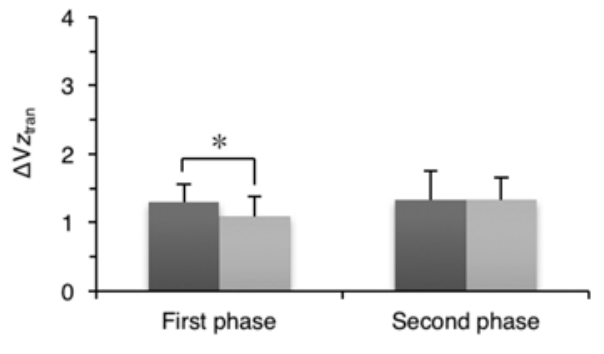

(f) $\Delta \mathrm{V} z_{\text {tran }}$

Figure 2. Means and standard deviation (SD) of the variance of segment angle that does not affect COM $\left(\mathrm{V}_{\mathrm{UCM}}\right)$, the variance that affects $\mathrm{COM}\left(\mathrm{V}_{\mathrm{ORT}}\right)$, and the synergy index $(\Delta \mathrm{V})$ in the horizontal and vertical directions after buttocks-off during STS in the older and younger group. Asterisks indicate significant differences between the older and younger groups $(* \mathrm{p}<0.05)$.

rection $\left(\mathrm{V}_{\mathrm{UCM}} z\right.$ and $\left.\mathrm{V}_{\mathrm{ORT}} z\right)$ for the first and second phases were higher in the older group than in the younger group. $\Delta \mathrm{V}_{\text {tran }}$ in the vertical direction $\left(\Delta \mathrm{V}_{\text {tran }}\right)$ for the first phase was lower in the older group than in the younger group (Fig. 2d-f).

\section{Discussion}

The purpose of this study was to investigate whether aging affects the motor coordination of joint movements required to achieve STS using the UCM analysis. $\Delta \mathrm{V}$ values in the vertical direction for the first phase were lower in the older adults than in the younger adults, which supported our hypothesis. However, $\Delta \mathrm{V}$ values in the horizontal direction for the second phase were higher in the older adults than in the younger adults, which did not support our hypothesis.

Previous studies on STS using the UCM analysis have adopted the head position, hand position ${ }^{6}$, ground reaction force ${ }^{11)}$, and linear and angular momentum of $\mathrm{COM}^{10)}$ as the performance variables. The STS is characterized by three essential components, namely, (a) generating sufficient joint torque needed to rise (progression), (b) ensuring stability by moving the COM from a large BOS to a narrow BOS defined solely by the feet (stability), and (c) the ability to modify movement strategies used to achieve these goals depending on environmental constraints (adaptation) ${ }^{3)}$. However, the STS maneuver performed in this study involved no change of environmental or task-related constraints, and thus did not require adaptive capacity. The STS consists of combinations of joint movements of the body and is provided hierarchically through the motor coordination of joint movements. The mechanism of the CNS focuses on the parameter related to the movement of motor points, rather than individual parameters such as muscles and joints ${ }^{23)}$. Moreover, COM is often considered the main objective of CNS control ${ }^{24)}$. Accordingly, the present study adopted the horizontal and vertical movements of COM as 
the performance variables and conducted the UCM analysis.

In the horizontal direction, the variance in $\mathrm{COM}$ in both phases showed no significant difference between the two groups, and ANOVA showed a significant main effect of variance component $\left(\mathrm{V}_{\mathrm{UCM}}>\mathrm{V}_{\mathrm{ORT}}\right)$ in both phases, indicating the presence of kinematic synergy. Control of the horizontal movement of COM after buttocks-off is the invariant feature during STS; this ensures that stability is maintained during vertical rise of the body ${ }^{25}$. Scholz et al. ${ }^{9}$ reported that the instantaneous the horizontal movement of COM was clearly stabilized by kinematic synergy reflected in much larger variance in directions that leave the COM unchanged relative to that in directions that affect COM. In addition, these effects were also stronger when standing up under challenging task constraints, such as when performing this maneuver on a narrow support surface. Previously, motor flexibility was found to be similar in younger and older adults when task demand was lower ${ }^{26}$. In the present study, $\mathrm{V}_{\text {Ucn }} y$ values in both phases were higher in the older group than in the younger group and $\Delta \mathrm{V} y_{\text {tan }}$ values for the second phase were higher in the older adults than in the younger adults. The variances of the hip and knee joint angles both phases and the ankle joint angle for first phase were higher in the older group than in the younger group. Since the variances of lower extremity joint angles during STS did not affect the control of the horizontal movement of COM in the older group, it is suggested that older adults employ the joint coordination pattern with good variance to stabilize the horizontal movement of COM after the buttocks-off.

In the vertical direction, variance in $\mathrm{COM}$ for the first phase was higher in the older group than in the younger group, and the ANOVA did not show a significant main effect of the variance component $\left(\mathrm{V}_{\mathrm{UCM}}>\mathrm{V}_{\text {ORT }}\right)$ in both phases, resulting in the absence of kinematic synergy. The first phase is required for simultaneous COM deceleration in the horizontal direction and COM acceleration in the vertical direction. Greve et al. ${ }^{11)}$ reported that older adults employed greater motor flexibility, stabilizing both horizontal and vertical movement of COM during STS. However, Scholz et al. ${ }^{9)}$ reported that joint movement showed no kinematic synergy stabilizing the vertical path of COM. In the present study, variance in the hip and knee joint angles in both phases and that in the ankle joint angle for the first phase were higher in the older group than in the younger group. Furthermore, the $\mathrm{V}_{\mathrm{UCM}}$ and $\mathrm{V}_{\mathrm{ORT}} \mathrm{z}$ in both phases were higher in the older group than in the younger group, and the $\mathrm{V}_{\mathrm{UCM}} z$ was smaller than $\mathrm{V}_{\text {ORT }}$ for for first phase. $\Delta \mathrm{V}_{\text {tran }}$ for the first phase was lower in the older group than in the younger group. From these results, we would surmise that variance in the joint angle of the lower extremities showed no kinematic synergy to stabilize the vertical movement of COM. This finding suggests that older adults may be unable to stabilize their vertical movement of COM after buttocks-off, as demonstrated by the absence of a kinematic synergy.

Our main findings suggest that older adults were able to maintain the coordination in the joint movements to stabilize the horizontal, but not vertical movement of COM. Younger adults can simultaneously perform the task of stabilizing the horizontal movement of COM after buttocksoff and the task of raising the vertical movement of COM during STS. However, as older adults require increasing levels of stability for the horizontal movement of COM after buttocks-off during STS, they may have decreased kinematic synergy for stabilization of the vertical movement of $\mathrm{COM}$ as a result. In fact, a failure to achieve STS might occur when the subject rises only slightly off the chair and then sits back down, due to either muscle weakness or balance control and coordination impairment ${ }^{1}$. In the present study, the older group comprised individuals with no impairment or disability who did not sit back down after attempting to rise. Given that even older individuals with high motor function need to focus on controlling the horizontal movement of COM during STS, with increased variance of the hip and knee angles after buttocks-off, less stabilization of the horizontal movement of COM might reflect the existence of impairment and failure in performing the STS.

There is a potential limitation to this study: the present findings pertain to highly active older adults registered at an employment service center and may not be applicable to patients with different symptoms and presentations of diseases. Further studies to evaluate movement coordination in sedentary or less active older adults who experience difficulties in performing STS would be valuable for the development of physical therapy interventions for older adults.

\section{Conclusion}

Older adults require increasing levels of stabilization of horizontal movement of COM after buttocks-off in the STS maneuver. As a result, variance in the joint angle of the lower extremities indicated no kinematic synergy for stabilizing the vertical movement of COM. The present study findings provide new insight into the elucidation of why some individuals experience difficulties in performing the STS maneuver.

Conflict of Interest: There is no conflict of interest to disclose.

Acknowledgements: This study was carried out at the Center for Advanced Practice and Research of Rehabilitation, Hiroshima University. We hereby express our sincere appreciation to Dr. Tetsushi Nonaka at Kobe University for his advice and guidance throughout this study. 


\section{References}

1) Riley PO, Krebs DE, et al.: Biomechanical analysis of failed sitto-stand. IEEE Trans Rehabil Eng. 1997; 5: 353-359.

2) Pai YC and Rogers MW: Control of body mass transfer as a function of speed of ascent in sit-to-stand. Med Sci Sports Exerc. 1990; 22: 378-384

3) Shumway-Cook A and Woollacott MH: Control of normal mobility. Chap 12. In: Motor Control. 4th ed, Lippincott Williams \& Wilkins, Philadelphia, 2007, pp. 315-347.

4) Alexander NB, Koester DJ, et al.: Chair design affects how older adults rise from a chair. J Am Geriatr Soc. 1996; 44: 356362.

5) Anan M, Okumura K, et al.: Effects of variation in cushion thickness on the sit-to-stand motion of elderly people. J Phys Ther Sci. 2008; 20: 51-57.

6) Scholz JP and Schöner G: The uncontrolled manifold concept: identifying control variables for a functional task. Exp Brain Res. 1999; 126: 289-306.

7) Scholz JP, Schöner G, et al: : Motor equivalent control of the center of mass in response to support surface perturbations. Exp Brain Res. 2007; 180: 163-179.

8) Latash ML, Krishnamoorthy V, et al.: Postural synergies and their development. Neural Plast. 2005; 12: 119-130.

9) Scholz JP, Reisman D, et al.: Effects of varying task constraints on solutions to joint coordination in a sit-to-stand task. Exp Brain Res. 2001; 141: 485-500.

10) Reisman DS, Scholz JP, et al.: Coordination underlying the control of whole body momentum during sit-to-stand. Gait Posture. 2002; 15: 45-55.

11) Greve C, Zijlstra W, et al.: Not all is lost: Old adults retain flexibility in motor behaviour during sit-to-stand. PLoS ONE. 2013; 8: e77760.

12) Hsu WL, Chou LS, et al.: Age-related changes in joint coordination during balance recovery. Age (Dordr). 2013; 35: 12991309

13) Verrel J, Lövdén M, et al:: Normal aging reduces motor syner- gies in manual pointing. Neurobiol Aging. 2012; 33: 200.e1-10.

14) Park J, Sun Y, et al.: Age-related changes in optimality and motor variability: an example of multifinger redundant tasks. Exp Brain Res. 2011; 212: 1-18.

15) Cohen J: A power primer. Psychol Bull. 1992; 112: 155-159.

16) Andriacchi TP, Galante JO, et al.: The influence of total kneereplacement design on walking and stair-climbing. J Bone Joint Surg Am. 1982; 64: 1328-1335.

17) Okada H, Ae M, et al.: Body segment inertia properties of Japanese elderly. Biomechanism. 1996; 13: 125-139 [in Japanese].

18) Black DP, Smith BA, et al.: Uncontrolled manifold analysis of segmental angle variability during walking: preadolescents with and without down syndrome. Exp Brain Res. 2007; 183: 511521.

19) Hsu WL and Scholz JP: Motor abundance supports multitasking while standing. Hum Mov Sci. 2012; 31: 844-862.

20) Winter DA: Antropometry. Chap 4. In: Biomechanics and motor control of human movement. 4th ed, John Wiley, Hoboken, 2009, pp. 82-106.

21) Krishnan V, Rosenblatt NJ, et al.: The effects of age on stabilization of the mediolateral trajectory of the swing foot. Gait Posture. 2013; 38: 923-928.

22) Robert T, Zatsiorsky VM, et al.: Multi-muscle synergies in an unusual postural task: quick shear force production. Exp Brain Res. 2008; 187: 237-253.

23) Latash ML: Multi-joint movement. Chap 21. In: Neurophysiological basis of movement, Human Kinetics, Champaign, 2008, pp. 231-240.

24) Winter DA: Human balance and posture control during standing and walking. Gait Posture. 1995; 3: 193-214.

25) Millington PJ, Myklebust BM, et al.: Biomechanical analysis of the sit-to-stand motion in elderly persons. Arch Phys Med Rehabil. 1992; 73: 609-617.

26) Hsu WL, Lin KH, et al.: Use of motor abundance in old adults in the regulation of a narrow-based stance. Eur J Appl Physiol. 2014; 114: 261-271. 